\title{
Perioperative Management of Budd Chiari Syndrome Patient on Oral Anticoagulant for Split Skin Graft Surgery
}

\author{
Authors \\ Satish Logidasan ${ }^{1}$, Gowri Shankar Anjineyan ${ }^{2}$, Kanimozhi Rathinasamy ${ }^{3}$ \\ ${ }^{1,2,3}$ Department of Anesthesiology, Govt Stanley Medical College, TN- 600001, India \\ Corresponding Author \\ Satish Logidasan
}

ABSTRACT

We discuss a case report of decompensated chronic liver disease (DCLD) on oral anticoagulant (Tab. warfarin) for hepatic vein thrombosis (BUDD CHIARI SYNDROME) optimised and taken up for incidental surgery. Preoperative optimisation of this chronic liver disease in a short span of time and Anaesthetic management for non hepatic surgery during the waiting period of liver transplantation is an unique challenge. Core Tip: The idea to present this case report is to emphasize the challenges in perioperative care of an anticoagulated Budd chiari syndrome liver disease patient who is posted for non hepatic incidental surgery.

Keywords: chronic liver disease with decompensation (DCLD), buddchiari syndrome, warfarin, non hepatic surgery, split skin graft [SSG], regional nerve block.

\section{Introduction}

Liver transplantation is the only effective survival option for DCLD patients, but our patient has a raw area foot which prevents him from being listed for liver transplantation. Many patients die when they are waiting for transplant, so it's a race against time to prepare and optimise his physiology for SSG surgery and then to add him to the waiting list for liver transplantation.

The common complications during surgery are due to severe coagulopathy, adult respiratory distress syndrome, spontanous bacterial peritonitis, encephalopathy, hepatorenal syndrome and sepsis. The degree of malnutrition, ascites, prothrombin time, concentration of serum albumin and serum bilirubin due to portal hypertension predicts the risk of complications and death following surgery.
Optimising Infections, Massive ascites, Anticoagulation for hepatic vein thrombosis, Lung at electasis, pleural effusion is a unique challenge. Purpose of this review is to discuss the preoperative optimisation strategies of anticoagulated DCLD patient and also about anaesthesia management for SSG surgery (Non-liver surgery) which is a challenging one for the Anaesthesiologist.

\section{Buddchiari Syndrome Patient}

A 22 yr male patient, Non diabetic, occasional alcoholic [weekly $180 \mathrm{ml}$ hard liqour ] and smoker [4-5 cig/day] for 6 years, presented with abdominal distention and bilateral pedal edema, caput medussae (inferior venacava syndrome). He was diagnosed as decompensated liver disease due to buddchiari syndrome with CHILD TOURCOTTE 
PUGH score $\mathrm{C}$ and MELD of 22 on regular liver transplant workup in our Institution. During his workup he sustained injury over right dorsum of foot, it started as a small ulcer and developed to $6 \mathrm{~cm} \times 6 \mathrm{~cm}$ size ulcer, margins well defined surrounded by edema, distal pulses were felt. Doppler came to be normal so as to rule out vascular cause. Plastic surgery team suggested split skin graft over the right dorsum of foot as an elective procedure so as to optimise infection before taken up for liver transplantation.
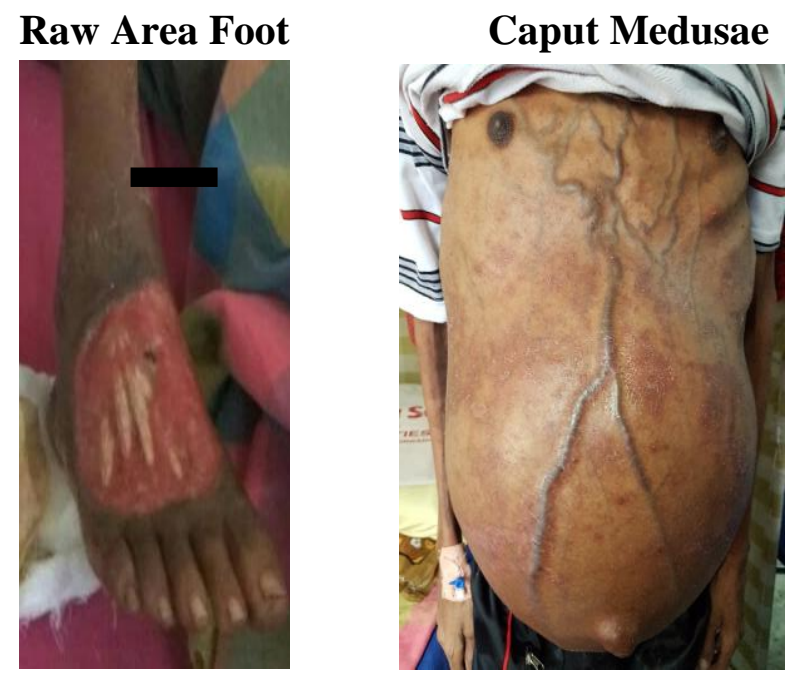

\section{Raw Area Foot}

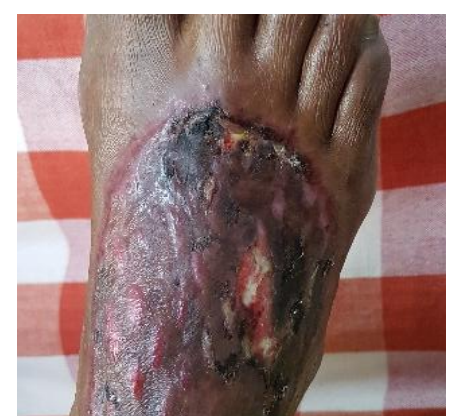

Abdominal imaging studies of this patient shows volume redistribution with patchy vascular congestion in liver and thrombosis of hepatic vein suggestive of Budd chiari syndrome. Modest narrowing of intrahepatic Inferior venacava (IVC syndrome). Liver is normal $15 \mathrm{~cm}$ size and shows reduced density, multiple ill defined homogenous nodules of varying sizes seen in both lobes of liver measuring 1-2 $\mathrm{cm}$. The nodules show moderate enhancement in both arterial and venous phase and washout in delayed phase.
Lung imaging showed bilateral pleural effusion linear at electatic changes in superior segments bilateral lower lobe and basal segment of right lower lobe. So Pulmonary function tests were done which revealed Restrictive pattern due to gross ascites.

\section{Pulmonary Function Test}

\begin{tabular}{|l|l|l|}
\hline FVC & 3.37 & $64 \%$ \\
\hline FEV1 & 2.44 & $55 \%$ \\
\hline \%FEV1 & 0.73 & $85 \%$ \\
\hline PEF & 6.44 & $69 \%$ \\
\hline $25-75$ & 1.81 & $36 \%$ \\
\hline MEF50 & 1.68 & $29 \%$ \\
\hline MEF25 & 0.96 & $36 \%$ \\
\hline
\end{tabular}

Brain imaging showed mild atrophic changes in bilateral cerebral hemispheres, few punctate $\mathrm{T} 2$ FLAIR hyperintensities in bilateral deep and subcortical white matter. Small vessel ischaemic changes were also present.

Echocardiogram showed no regional wall motion abnormality, normal systolic function with ejection fraction of $52 \%$ and bubble contrast study showed no crossover so as to rule out intrapulmonary vascular shunts and dilatation.

\section{Optimisation Strategy Followed For This Patient}

Anticoagulation on warfarin

Massive ascites

Infections

Control Severe portal hypertension

Prevention of hepatic encephalopathy

Respiratory care for atelectasis and pleural effusion

Bridging therapy for surgery

Nutrition management

Patient had thrombosis of hepatic vein, so he was started on tab. acitrom (warfarin) $1 \mathrm{mg}$ od and titrated so that INR was maintained between 2.0-2.5. Tab. Warfarin was stopped 5 days before the planned procedure. He was started on bridging therapy of LMWH $0.4 \mathrm{mg}$, stopped $24 \mathrm{hrs}$ before the procedure.

Massive ascites caused respiratory distress and was planned for paracentesis with $10 \mathrm{~g}$ of $20 \%$ albumin per liter of ascitis drained. Salt restricted to $2 \mathrm{~g} / \mathrm{day}$, spironolactone (aldactone) $100 \mathrm{mg}$ twice daily, 
frusemide $40 \mathrm{mg}$ twice daily were started. His ascitic fluid culture showed polymorphonuclear cells 400 cells/mm3 and diagnosed to have spontaneous bacterial peritonitis, so inj.Cefotaxime $1 \mathrm{gm}$ thrice daily, Tab. Norfloxacin 400mg twice daily and albumin were started. Throat swab showed enterococci and nasal swab showed coagulase negative streptococci which was sensitive to inj. Amikacin and inj.Gentamicin, and he was on antibiotics for one week. Tab. Propranolol 40mg given for portal hypertension.

Chest physiotherapy, Nebulisation, Triflow exercises were encouraged.

After assessing nutritional status by Subjective global assessment,his calorie requirement calculated and he received $1.2 \mathrm{~g}$ protein $/ \mathrm{kg} /$ day and 30 $\mathrm{kcal} / \mathrm{kg} / \mathrm{day}$.

\section{Lab Investigation}

\section{Pre-Optimisation}

\begin{tabular}{|c|c|c|c|}
\hline Platelets & 86000 & Platelets & $1,15,000$ \\
\hline Hemoglobin & 10.8 & Hemoglobin & 11.2 \\
\hline Total Count & 17500 & Total Count & 8500 \\
\hline INR & 3.8 & Polymorphs & 2.4 \\
\hline RBS & 124 & RBS & 112 \\
\hline Urea & 43 & Urea & 46 \\
\hline Creatinine & 1.63 & Creatinine & 1.12 \\
\hline Total Bilirubin & 12.5 & Total Bilirubin & 11.5 \\
\hline Direct Bilirubin & 10.6 & Direct Bilirubin & 9.5 \\
\hline SGOT/SGPT & $225 / 189$ & SGOT/SGPT & $223 / 179$ \\
\hline Albumin & 2.8 & Albumin & 3.4 \\
\hline Na+/K+ & $141 / 3.5$ & Na+/K+ & $134 / 3.8$ \\
\hline
\end{tabular}

After optimisation we planned for split skin graft as a elective surgery under peripheral nerve blockade (femoral and sciatic nerve block) for right leg with ultrasound guidance. Preoperative vital parameters were stable. Antibiotic Inj.Cefotaxime $1 \mathrm{gm}$ given before starting the surgery. Aseptic precaution undertaken. Local anaesthetic $0.25 \%$ bupivacaine $40 \mathrm{ml}$ and inj adrenaline $2 \mathrm{mcg} / \mathrm{ml}$ solution prepared for peripheral nerve block were used.

Block onset: 8 min

Fluid: Normal saline $500 \mathrm{ml}$ and dextrose $25 \%$ as infusion(to prevent hypoglycaemia)

Sedation: Fentanyl $20 \mathrm{mcg}$

Vitals signs: PR 77-85/min., BP 108-122/56-71mm hg.,Spo2 99-100\%
Sugars: $92 \mathrm{mg} / \mathrm{dl}$ (capillary)

Temp:36.8 C

Oxygenation : oxygen mask $61 / \mathrm{min}$.

Intraoperative period was uneventful and the Surgery duration was one and half hours. He was shifted to surgical ICU for monitoring. Post operative PR 72/min BP 112/64mm hg SPO2 at room air $96 \%$. Postoperative Analgesia lasted for 10 hrs, he required inj. fentanyl $20 \mathrm{mcg}$ [three aliqouts] as rescue analgesia.

\section{Discussion}

Estimating Operative Risk In Patients With Liver Disease
A. Child Tourcotte Pugh Score (CTP)
B. Meld
C. American Society of Anaesthesiologist-Physical Status

D. Apache Scoring System

In Cirrhotic patients, Teh et al documented that an American Society of Anaesthesiologists (ASA) class of IV added the equivalent of 5.5 Model for End Stage Liver Disease (MELD) points to the mortality rate, whereas an ASA class of $\mathrm{V}$ was associated with a $100 \%$ mortality rate. The influence of the ASA class was greatest in the first 7 days after surgery, after which the MELD score became the principal determinant of outcome. The risk of postoperative mortality and morbidity correlate(s) well with the categorization of the patient as per the Child-Turcotte-Pugh (CTP) class of cirrhosis. In a retrospective analysis (from 1992 to 1999) of 40 patients with cirrhosis who underwent non-hepatic surgical procedures, the presence of tense ascites, low albumin value, deranged prothrombin time, activated partial thromboplastin time, together with the emergency of the operation, was significantly correlated with a mortality of $7.1 \%$ in Child's class A, of $23 \%$ in class $\mathrm{B}$, and of $84 \%$ in class C. MELD scoring system MELD score is utilized to prioritize organ allocation to the probable liver transplant recipients. The MELD score is considered objective and reliable because it is based on objective criteria, i.e., serum bilirubin, serum creatinine and international 
normalized ratio (INR). The score can be calculated by an online MELD calculator like the one at www.unos.org/resources. $(1,2)$

CTP score 5-6 CTP A <5\% mortality risk

CTP score 7-9 CTP B 25\%-50\%

CTP score10-15 CTP C > 50\%

\begin{tabular}{|c|c|}
\hline PREDICTOR OF COMPLICATIONS & PREDICTOR OF MORTALITY \\
\hline Child-pugh class $\mathrm{B}$ and $\mathrm{C}$ & Male gender \\
\hline Ascites & Child-Pugh class B and C \\
\hline Etiology of cirrhosis other than primary biliary cirrhosis & Etiology of cirrhosis other than primary biliary \\
\hline Elevated creatinine Ascites & cirrhosis \\
\hline Preoperative infection & Ascites \\
\hline Chronic obstructive pulmonary disease & Preoperative infection \\
\hline Preoperative upper gastrointestinal bleed & Respiratory surgery \\
\hline Invasiveness of surgical procedure & ASA IV V \\
\hline Intraoperative hypotension ( $20 \%$ decrease of base line & \\
\hline ASA IV V & \\
\hline
\end{tabular}

\begin{tabular}{|l|l|l|}
\hline Preoperative variables & $\begin{array}{l}\text { Percent of } \\
\text { mortality if } \\
\text { factors present }\end{array}$ & $\begin{array}{l}\text { Percent of } \\
\text { mortality if } \\
\text { factors absent }\end{array}$ \\
\hline Child class & & \\
A & 10 & 11 \\
B & 31 & 10 \\
C & 76 & 17 \\
Ascites & 58 & 12 \\
Emergency surgery & 57 & 18 \\
Bilirubin $>3$ mg/dL & 62 & 19 \\
Albumin $<3$ mg/dL & 58 & \\
Prothrombin time $>1.5$ s above control & 63 & \\
White blood cell count $>10000$ & 54 & \\
P $<0.01$ for all variables & & \\
\hline
\end{tabular}

MELD $<10 \rightarrow$ may undergo elective procedures

MELD 10-15 $\rightarrow$ Elective surgery may be done with the potential of surgery related side effects.

MELD $>15$ who is a candidate for liver transplantation elective surgery should be avoided.

In hospital mortality rates after various nontransplant surgical procedures-

$8.3 \%-25 \%$ compared to $1.1 \%$ (non- cirrhotic)

\section{APACHE scoring system}

The Acute Physiology, Age and Chronic Health Evaluation System (APACHE III) score can predict survival in cirrhotic patients admitted to an intensive care unit. Cirrhotic patients admitted to the medical intensive care unit are associated with high mortality rates. While both Child-Pugh and the APACHE Iscores can satisfactorily predict the outcomes for critically ill cirrhotic patients,
APACHE $I$ is more powerful in discriminating the survivors from the non-survivors after non -liver surgeries.

\section{PREDICTOR OF MORTALITY}

Child-Pugh class B and C

Etiology of cirrhosis other than primary biliary

Preoperative infection

Respiratory surgery

ASA IV V

\section{Ascites \& Anaesthesiologist}

In a study by Conn, ascites in patients with cirrhosis was associated with a $37-83 \%$ mortality rate compared with $11-53 \%$ in those without ascites. Ascites needs to be assessed and managed before surgery because it can lead to abdominal wall herniation, and respiratory compromise secondary to reduced lung expansion.. In general, ascites should be treated aggressively with diuretics and/or large-volume paracentesis before surgery.

Ascites fluid can also be removed intraoperatively at laparotomy. It is important to take note of the volume of fluid removed and the patient's baseline renal function and to consider albumin replacement to maintain intravascular volume and prevent paracentesis-induced circulatory dysfunction.

It was very difficult to position the patient because of enormous abdomen and peripheral nerve 
blockade of the leg becomes unsuccessful, without prior clinical optimisation, and the anaesthetic technique would become very difficult leading to potential complications. ${ }^{(6)}$

\section{Malnutrition \& Anaesthesiologist}

Liver has a central role in nutritional homeostasis and any liver disease leads to abnormalities in nutrient metabolism and subsequent malnutrition. A variety of assessments can be used to evaluate patients' nutritional status. Anthropometric tools include BMI, midarm muscle circumference, and triceps skin fold thickness. Functional tests-such as hand-grip strength - can assess muscle strength as a marker of muscle tone. Finally, subjective global assessment tools, which are typically in the format of flow charts or algorithms, can integrate information about patients' dietary intake, weight loss history, gastrointestinal symptoms, functional capacity, duration and degree of abnormal intake.

Since malnutrition affects post-transplant survival, precise assessment must be an integral part of preand post-surgical management. While there is wide agreement that aggressive treatment of nutritional deficiencies is required, strong scientific evidence supporting nutritional therapy is sparse. In practice, oral nutritional supplements are preferred over parenteral nutrition, but enteral tube feeding may be necessary to maintain adequate calorie intake. Protein restriction should be avoided and administration of branched-chain amino acids may help yield a sufficient protein supply

In 2009, European Society for Clinical Nutrition and Metabolism, published guidelines specifically addressing patients with liver disease, these guidelines advise that patients with liver disease consume $35-40 \mathrm{kcals} / \mathrm{kg} / \mathrm{day}$, with a protein intake of $1.2-1.5 \mathrm{~g} / \mathrm{kg} / \mathrm{day} .^{(11)}$

\section{Spontaneous Bacterial Peritonitis}

Patients with ascitic fluid polymorphonuclear leukocyte (PMNL) counts of 250 cells per mm3 or greater should receive empiric antibiotic therapy (e.g., Cefotaxime $2 \mathrm{~g}$ intravenously every eight hours) and albumin (1.5 g / kg). Oral Ofloxacin (400 mg twice daily) is an alternative to intravenous medications in patients without vomiting, shock, severe hepatic encephalopathy, or a creatinine level greater than $3 \mathrm{mg}$ per $\mathrm{dL}$. Patients with gastrointestinal hemorrhage and cirrhosis should receive Norfloxacin or Trimethoprim/ sulfamethoxazole twice daily for seven days. The organisms seen in this group were Gram-positive and the patient survival at 1 yr was $67 \%$.Hospitalized patients are at risk of more serious infection with SBP, usually with Gramnegative organisms and with a much higher mortality rate. $46 \%$ in the series reported by Lipka et al. Predominant organisms were Escherichia coli and Klebsiellapneumoniae. ${ }^{(9,10)}$

\section{Why General Anaesthesia Avoided}

General anesthesia reduces total hepatic blood flow, especially the contribution of the hepatic artery. Patients with liver disease tend to have several baseline cardiovascular abnormalities, including decreased systemic vascular resistance and increased cardiac index, which may further affect hepatic blood flow. In addition, catecholamine and other neurohormonal responses are impaired in patients with liver disease; therefore, intraoperative hypovolemia or hemorrhage may not trigger adequate compensatory mechanisms. Anesthetics further blunt this response. The result of this reduction in hepatic perfusion is a drastic loss of their remaining marginal hepatic function.

Decreased synthesis of plasma-binding proteins and hypoalbuminemia impairs drug binding and metabolism and elevates serum drug levels. Impaired drug metabolism, detoxification, and excretion by the liver can prolong drug half-lives.

Of all the inhaled anesthetics, halothane and enflurane appear to reduce hepatic artery blood flow the most because of systemic vasodilation and a mild negative inotropic effect. Halothane is also associated with the greatest risk of hepatotoxicity, with the incidence of fulminant hepatitis approx imating 1 case in 6,000-35,000 patients after exposure. 
Studies showed that advanced hepatic disease does not significantly affect the pharmacokinetics of Vecuronium. Atracurium has a theoretical advantage because its metabolism is not dependent to hepatic function. So, clearance and elimination half-life of Atracurium in patients with impaired hepatic or renal function is not particularly different from those who have normal hepatorenal function. However, it has been found that because of larger volume of distribution, distribution half-lives are shorter in patients with severe hepatorenal dysfunction compared to normal individuals. Careful injection of any muscle relaxant according to transcutaneous nerve stimulator monitoring is beneficial. Likewise, drugs such as morphine, meperidine, benzodiazepines, and barbiturates should be used with caution because of their dependence on the liver for metabolism. In general, the doses of these agents should be decreased by $50 \%$. Fentanyl is considered the opioid of choice in these patients because when used in relatively moderate doses, it does not decrease hepatic oxygen and blood supply, nor does it prevent increases in hepatic oxygen requirements .

\section{Why Regional Anaesthesia Preferred Over General Anaesthesia}

Whenever possible, regional anesthesia might be used in patients with advanced liver disease. This lowers the possibility of loss of consciousness and delayed recovery due to difficulty in drug metabolism as in general anaesthesia. Both neuraxial and regional nerve block anesthesia could be considered in patients with hepatic failure. Regional anesthesia attenuates surgery-induced stress responses, such as increase in levels of corticosteroid hormone and catecholamine. Regardless of the effects of stress hormones on hemodynamic and circulation, they are thought to play an important role in depressing immune function .In all cases under anesthesia, arterial blood pressure should be preserved and sympathetic stimulation avoided. Total consumption dose while performing regional anesthesia should be cautiously calculated and close monitoring for any possible side effects is necessary. Coagulopathy should be considered as a contraindication to some types of regional anesthesia. However, can be considered with acceptable coagulation profile.

\section{Why Spinal Anaesthesia Avoided}

Performing epidural or spinal anesthesia in patients treated with anticoagulation drugs is clearly a concern. The occurrence of bleeding in an enclosed space, such as the spinal canal, without the normal protective mechanisms of the coagulation system may cause major neurologic damage. However, major nerve blocking techniques can be used in some patients who have received or will be receiving anticoagulation drugs. The correct approach to this dilemma cannot be dogmatic but must be a graded one. Knowledge of the pharmacologic properties of the different anticoagulants and their combination with strict patient selection, individual risk-benefit analysis, appropriate regional anesthetic techniques, respecting proper time intervals separating anticoagulant and epidural or spinal anesthesia, and continuous awareness of the possibility that a compressing spinal hematoma may develop should enable us to make anesthetic practice safer without withholding anesthetic techniques from patients who would most certainly benefit from them.

\section{Why Peripheral Nerve Block Preferred Over Spinal Anaesthesia}

- Analgesic efficacy

- Low risk of complication

- Tissue perfusion

Combined femoral and sciatic nerve block provides longer duration of postoperative analgesia of about 12-13 h as compared to a central neuraxial block of about $4-5 \mathrm{~h}$ and gives adequate pain control resulting in early mobilization. This had the beneficial effect of the use of less number of analgesic doses in the form of nonsteroidal antiinflammatory drugs and opioids which have many side effects such as postoperative nausea, vomiting, sedation, then leading on to possible drug pharmacokinetics, The posterior approach of Labat 
had better success rates as compared to other approaches. Similar results recorded by Tagariello et al, in a study on sciatic nerve block approaches showed that success rate with the posterior approach was found to be almost $99 \%$ over 15,000 patients.

\section{Why Ultrasound Preferred Over Nerve Stimulator For PNB}

Less incidence of neurovascular lesion

Direct visualisation of peripheral nerves, needle, the adjascent structures

Controlled distribution of the local agents

We observed that the use of USG in the conduction of interscalene brachial plexus block was associated with statistically significant shorter procedural and onset times than NS. They achieved high procedural success rates and high patient satisfaction scores, with low postoperative neurologic complications, when using ultrasound guidance for nerve blocks.

Investigations of large scale randomised controlled trials studying regional anaesthesia in conjuction with coagulation altering medications are not feasible due to medico legal consideration since nerve tissue compromise from hematoma development is rare, very large sample size are reported. Therefore Attempts at striking a balance between catastrophic thromboembolic events and hemorrhagic complication will remain a strategy for clinician practising regional anaesthesia in the perioperative environment.

\section{Anticoagulation and Bridging Therapy}

Medical therapy is oriented toward preventing further progression of the venous thrombosis, and treating the underlying prothrombotic condition. Anticoagulation should be administered to patients with BCS.

Anticoagulation alone is sufficient in only $10 \%$ of patients, especially those with mild disease. There are no data to suggest a difference in efficacy of glycosaminoglycans (unfractionated heparin or lowmolecular weight heparin (LMWH)) as compared to vitamin $\mathrm{K}$ antagonists. An alarmingly high rate of heparin induced thrombocytopenia (HIT) has been reported with unfractionated heparin in BCS as compared to other indications (12.3\%, compared with $2.7 \%$, respectively). LMWH should be started promptly in a patient with a new diagnosis of BCS because of its rapid method of action.Vitamin $\mathrm{K}$ antagonists should be initiated when deemed clinically desirable (no need for quick reversal of the anticoagulant effect) with an INR goal between 2 and 3 .

\section{Conclusion}

Anesthesia in chronic liver disease is a challenging condition for every anesthesiologist, which deserves meticulous attention in optimizing the patient's condition preoperatively and choosing appropriate anesthetic regimen and drugs. Optimising before a elective procedure in a short span of time is crucial to minimise post operative complications. control of infection and anticoagulation protocol before a regional peripheral nerve block is mandatory.

\section{References}

1. Anesthesia for Patients With Liver Disease Poupak Rahimzadeh 1; Saeid Safari 1,2; Seyed Hamid Reza Faiz 1; Seyed Moayed Alavian 2,3,* Hepat Mon. 2014 July; 14(7): e19881. DOI: 10.5812/hepatmon.19881 Published online 2014 July 1.

2. Assessment of risk for non-hepatic surgery in cirrhotic patients Prashant Bhanguil, Alexis Laurent2, Roland Amathieu3, Daniel Azoulay4, Europian association of study of liver .journal of hepatology.

3. revcolombanestesiol . 2010 5;4 3(3):250253 Revista Colombiana de Anestesiología Colombian Journal of Anesthesiologyw w w.revcolanest.com.co Case report Importance of sympathectomy induced by continuous brachial plexus block for digital replantation in a patient with liver cirrhosis: Case report - Rosana Guerrero-Domíngueza,*, Daniel López-Herrera-Rodrígueza, Guiomar Fernández-Castelllanob, Francisco Federeroa, Ignacio Jiméneza

4. Journal of Clinical and Experimental Hepatology Surgery in a Patient with Liver 
Disease Rakesh Rai*, Sanjay Nagral**, AabhaNagraly *Department of HPB Surgery and Liver Transplantation, Fortis Hospital, Mulund, **Department of Surgical Gastroenterology, Jaslok Hospital, and Department of Gastroenterology, Jaslok Hospital, Mumbai, India

5. Assessment of risk for non-hepatic surgery in cirrhotic patients Prashant Bhanguil, Alexis Laurent2, Roland Amathieu3, Daniel Azoulay4, $\Uparrow$

6. Caput Medusae In Alcoholic Liver Disease Case Report *Konrad Wroński1, 2 1Faculty of Medicine, Department of Oncology, University of Warmia and Mazury, Olsztyn, Poland Head of Department: prof. SergiuszNawrocki, MD, PhD 2Department of Surgical Oncology, Hospital Ministry of Internal Affairs with Warmia and Mazury Oncology Centre, Olsztyn, Poland Head of Department: AndrzejLachowski, MD

7. Local and Regional Anesthesia Neuraxial and peripheral nerve blocks in patients taking anticoagulant or thromboprophylactic drugs: challenges and solutions Jinlei $\mathrm{Li}$ Thomas Halaszynski Department of Anesthesiology, Yale University, Yale New Haven Hospital, New Haven, CT, USA

8. World J GastrointestSurg2012 December 27; 4(12): 267-274 ISSN 1948-9366 (online) Perioperative risk factors in patients with liver disease undergoing non-hepatic surgery

9. Preoperative preparation of patients with advanced liver disease Richard A. Wiklund, MD

10. Cirrhosis and Chronic Liver Failure: Part II. Complications and Treatment Joel J. Heidelbaugh, M.D., And Maryann Sherbondy, M.D. University of Michigan Medical School, Ann Arbor, Michigan

11. https://www.researchgate.net/publication/45 536019_Nutrition_Support_in_End-

Stage_Liver_Disease
12. Anesth Analg 1994;79 11 65-77 Review Articles Vandermeulen Et Al. 1175 Anticoagulants And Spinal-Epidural Anesthesia.

13. World J GastrointestSurg2012 December 27; 4(12): 267-274 